\title{
Das Kreuz mit dem Game
}

\section{Warum die Religion im Videospiel stärkere Beachtung verdient. Von Stefan Piasecki}

\begin{abstract}
Videospiele sind traditionell Orte voller Mythen, Rätsel, Sagen und Religion. Erste Spiele stellen Spieler vor moralische Entscheidungen. Inhalte und Erfahrungsrahmen ergeben sich nicht mehr durch vordefinierte Skripte sowie technische Bedingungen und Begrenzungen, sie sind immer mehr das Ergebnis freier menschlicher Entscheidungen und Handlungen in ansonsten zunehmend umfangreichen Spielwelten. Die traditionellen Begrenzungen zwischen (fiktivem) Spiel und (realer) Lebenswelt verschwimmen. So gut wie alle technischen Geräte erlauben heute eine Form von Spiel. Computer- oder Videospiele melden sich selbsttätig zurück und informieren beispielsweise über das Smartphone über gegenwärtige Vorgänge in der Spielwelt. Wenn Spiele also in die Lebenswelt übergreifen und Verhalten und Denken beeinflussen können, ist es an der Zeit zu berücksichtigen, wer eigentlich mit welchen Intentionen diese Spiele entwickelt. In einer 2012 an der Berliner Games Academy durchgeführten Studie wurden erstmals Spieleentwicklerinnen und -entwickler nach ihren weltanschaulichen Ansichten befragt.
\end{abstract}

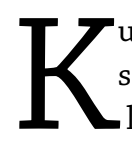
ultur, Religion und Identität - Begriffe, die vielfältige Assoziationen auslösen und insbesondere in gesellschaftraussetzen, treffen auf ein Medium, das sich sowohl technologisch wie auch in seiner gesellschaftlich und zivilisatorischen Bedeutung in den letzten dreißig Jahren erheblich entwickelt und verändert hat und dessen Produkte sich in unterschiedliche Kategorien wie Actionspiele, Rennspiele, Denkspiele aber auch Gruppen- oder Spiele in sozialen Netzwerken einteilen lassen. ${ }^{1}$

1 Übersicht zur kategorialen Einordnung von Spielegenres auf der Website der Unterhaltungssoftware Selbstkontrolle (USK): http://www.usk.del pruefuerfahren/genres/(zuletzt aufgerufen am 14.3.2014).
Prof. Dr. Stefan

Piasecki ist Professor für Handlungsfelder der Sozialen Arbeit an der CVJM-Hochschule in Kassel. Er ist außerdem Jugendschutzprüfer bei der FSK in Wiesbaden. 
Ein Videospiel oder Computerspiel ist also die Art von Spiel, bei der wesentliche Informationszusammenhänge und Handlungsbegründungen auf der Basis von auf Bildschirmen verfügbar gemachten Daten erfolgen. Der Hintergrund ist hierbei nicht real - auch wenn in Simulationen Flugzeuge geflogen oder chirurgische Operationen durchgeführt werden können. Gerade Videospiele machen den technischen Fortschritt im Bereich der visuellen und virtuellen Mensch-Maschine-Interaktion anschaulich. Strategiespiele und Simulationen für Heimcomputer bieten heute bereits einen Umfang und eine Handlungsbreite, die vor zehn Jahren mancher Militärrechner nicht erreicht hätte. $^{2}$

Ob spielerisches Spiel oder simulierte Realität: In virtuellen und künstlichen Welten können sich Spieler unbekannten Situationen aussetzen und ihnen ohne Angst begegnen. Während noch vor wenigen Jahren Videospielende vornehmlich jung waren, argwöhnisch beäugt von Eltern und Erziehungsberechtigten, pädagogisch, wissenschaftlich und politisch Tätigen, werden zunehmend Ältere aktiv (vgl. Dogruel 2008, S. 32ff.) und es steigt die Zahl von Untersuchungen zur Rezeption, Herstellung und Bewertung von Computer- und Videospielen. Methodentheoretische Erkenntnisse sowie rezeptionspsychologische Theorien und Verfahren aus der Medienforschung finden auch im Videospielbereich Berücksichtigung.

Neben den Massenmedien, die sich noch häufig primär für Gewaltaspekte interessieren (vgl. Bornhöft et al. 2009), beschäftigt sich eine zunehmende Zahl von differenzierten und wissenschaftlichen Untersuchungen mit pädagogischen, didaktischen oder sozialwissenschaftlichen (vgl. Meister et al. 2008) und auch theologischen Fragestellungen (vgl. Dinter 2007, S. 371).

Das Forschungsinteresse reicht hier von der Rolle der Gewalt im Spiel über die Wirkung der Transferprozesse und kognitiven Verarbeitung der Inhalte bis hin zu Einsatzmöglichkeiten im Schulunterricht (vgl. Brenner 2003; Waltemathe 2011). Neuerdings mehren sich auch Studien zur Frage der Politikvermittlung und -darstellung im Videospiel (vgl. Bevc 2007).

2 So wird ein Netzwerk von 16 Playstation-3-Spielekonsolen in einem Projekt der Universitäten von Alabama (Huntsville) und Massachusetts (Dartmouth) zur Berechnung der Schwerkraft sich vereinigender schwarzer Löcher genutzt: http://gravity.phy.umassd.edu/ps3.html (zuletzt aufgerufen am 14.3.2014). 
Ein bislang weitgehend vernachlässigter Bereich ist die Frage religiöser Implikationen. Nur eine Handvoll Artikel, einige größere Aufsätze und wenige gebundene Publikationen (vgl. Nord 2008, S. 19ff.; Reinders 2006, S. 47) widmen sich bislang im deutschsprachigen Raum diesem Komplex (vgl. Konz 2005, S. 237ff.; Winklmann 2014, S. 52ff.). Die großen Kirchen bleiben verhalten - namhafte und interessante Initiativen gibt es eher von unten, auf der Ebene lokaler Kirchenkreise, des Christlichen Vereins Junger Menschen (CVJM) oder von kirchennahen Einrichtungen mit einem Schwerpunkt im Kampf gegen „Internet-Sucht“ 3 (vgl. Alexander 2013; Kreusing 2014). Dabei wäre gerade angesichts drängender Fragen der religiösen Verortung in einer säkularisierten und mediatisierten Gesellschaft (vgl. Keppler 2010, S. 111ff.) eine direktere Beschäftigung von Kirchen mit dem Medium Videospiel denkbar, und es sollte die Kirchen interessieren, was gerade Jugendliche in den virtuellen Mythenwelten des Videospiels finden, was sie in den MyEs sollte die Kirchen interessieren, was Jugendliche in den virtuellen Mythenwelten des Videospiels finden, thenwelten der Kirche nicht mehr zu suchen was sie in der Kirche nicht mehr suchen. wagen (oder zu finden hoffen). ${ }^{4}$ Wollen sie lediglich Langeweile entfliehen, können sie eine Alltagsleere nicht mehr ertragen, wollen sie das „Nichts“ (Baudrillard 2000, S. 14) abschaffen? Sinkt durch eine immer höhere Spezialisierung der intersubjektiven Alltagswelt, die allen gemeinsam gehört, mit denen das Subjekt vielfältig verbunden ist (vgl. Waltemathe 2011, S. 60), die Freiheit des Einzelnen so stark, dass Menschen Freiraum nun in virtuellen Bereichen suchen, wo sie diesen vermeintlich eigenmächtig bestimmen und kontrollieren können?

Jugendliche sind durchaus auf der Suche nach weltanschaulicher Orientierung, und gerade sie sind nach Freudenberger-Lötz auf eine pädagogische Begleitung angewiesen und auch an ihr interessiert (vgl. 2012, S. 261f.). Videospiele sind nicht nur voll von religiöser Symbolik, sie erlösen auch (von der Realität) und berufen zu etwas (einer hehren Aufgabe, einer Suche etc.): Das Ich des Spielers oder der Spielerin erlebt sich herausgenommen aus einer sterilen Realität etwa der Ausbildungs- oder Berufszwänge und

3 Siehe zum Beispiel die Initiative "Return“ der „Fachstelle für exzessiven Medienkonsum", einer christlichen Drogenhilfe und Mitglied im Diakonischen Werk der evangelisch-lutherischen Landeskirche Hannover: http:// www.return-mediensucht.de/ (zuletzt aufgerufen am 11.9.2014).

4 Grethlein spricht von gemeindlichen "Binnenräumen“, zu denen immer weniger Menschen Zugang haben (Grethlein 1998, S. 535). 
betreibt seine Transformation in eine grundsätzlich beherrschbare (virtuelle) Welt, in der das eigene Tun zudem weitgehend folgenlos bleibt - zumindest für realweltliche Bezüge.

Hinzu kommt: In Spielen finden sich gehäuft religiöse Bilder und Bekenntnisse, wie es sie in der Realität so eindeutig kaum noch gibt - sie verlangen nach Stellungnahme, Akzeptanz und Befolgung bzw. Unterwerfung, durch die alleine man

In Videospielen finden sich gehäuft religiöse Bilder und Bekenntnisse, wie es sie in der Realität so eindeutig kaum noch gibt. als Spielender Teil der exklusiven (virtuellen) Wirklichkeit werden und bleiben kann. WortBotschaften und unumstößliche Leitlinien, die in der Realwelt massiver Kritik ausgesetzt wären, gelten in Videospielen als normal und werden hingenommen. Medienpädagogik mit Videospielen kann also durchaus zum Kernbereich religiöser Bildung gezählt werden (vgl. Pirner 2012b, S. 169).

Während Religion im Jugendbuch, im Film und auch in der Popmusik vielfach und umfangreich untersucht wurde, blieben Computer- und Videospiele bislang meistens außen vor. Gerade vor diesem Hintergrund ist bedeutend, was Böhm und Buschmann über die popularkulturelle Bedeutung der Popmusik für die Religionsvermittlung festgestellt haben: „Eine subjekt-sensible Religionspädagogik, orientiert an den Perspektiven von Kindern und Jugendlichen, muss sich an den Quellen orientieren, die primär Kinder- und Jugendperspektiven haben: ihre Lebenswelten“ (Böhm/Buschmann 2000, S.12). Hierzu gehören auch prägende mediale Phänomene wie „Video, Computer, Fantasy und ScienceFiction-Welten, Graffiti, Werbung usw.“ (ebd.).

Wenn jedoch rezeptionsanalytische Untersuchungen einzelne Videospiele und ihre Sujets aufgreifen, die älter als zwei oder drei Jahre sind, bilden sie angesichts des technischen Fortschritts stets nur eine rapide verblassende Vergangenheit ab. Einzig eine kontinuierliche Begleitforschung kann hier Abhilfe schaffen und die Muster des Marktes sowie die gestalterische Webart der Spiele und die Intentionen der Entwickler als Weltenerschaffer sichtbar machen.

$\mathrm{Zu}$ berücksichtigen ist auch, wie Spielerinnen und Spielern der Zugang gestaltet wird: Spielekonsolen der neuesten Generation (wie jüngst die Playstation 4 oder Xbox One) bieten zwar bis dahin ungekannte grafische Plastizität und physikalische Gesetzmäßigkeiten wie Wind und Wetter, die ständig die Spielwelt verändern und Spieler zur Anpassung des eigenen Handelns zwingen. Neuartige Steuerungseinheiten (Joypads), die auch die Bewegungen des Spielers im 360-Grad-Raum aufnehmen (Nin- 
tendo Wii) oder die Bewegungen der Spieler und ihre Umgebung beobachten (Microsoft Xbox One) und die so gewonnenen Daten in das Spiel integrieren, lassen die schlichte Zweidimensionalität der TV-Röhren vergangener Zeiten vergessen. Der neuartige Begriff der „Exergames“ bezeichnet Spiele, „bei denen der Spieler sich bewegen muss, um etwas zu erreichen“ (Kopka 2013, S. 265).

Bereits diese technische Evolution unterwirft aber wissenschaftliche Erkenntnisse bezüglich älterer Spiele und ihrer Einflüsse auf Spieler fast einer ebenso ultra-kurzen Verwertungszeit wie die Spiele selbst, die in den allermeisten Fällen nach nur wenigen Monaten am Markt durchverkauft sind. Untersuchungen, die fünf oder sogar zehn Jahre alt sind und deren Ergebnisse sich auf noch ältere Spiele beziehen oder als Referenzen noch ältere Literatur heranziehen, sind schwer weiterhin zu berücksichtigen.

Gerade weil Religion im gesellschaftlichen Kontext eine nachhaltig hohe Bedeutung und das Potential für interkulturelle Verständigung ebenso wie für Missverständnisse besitzt und weil Videospiele ein milieuübergreifendes, internationales, die Generationen und Kulturen verbindendes Massenphänomen mit hohem Attraktivitätswert bleiben, sollte nach grundsätzlichen Erscheinungsmustern von Religion im Spiel gefragt werden und danach, wie in Spielen bereits heute religiöse Elemente eingesetzt werden oder diese zukünftig Eingang in die interaktive Unterhaltung finden könnten.

\section{Narration: Mythen, Sagen und Legenden im digitalen Kosmos}

Für die narrative Ebene digitaler und interaktiver Unterhaltungsprodukte sind nicht in erster Linie die technischen Daten oder die grafische Auflösung von Bedeutung, sondern Dramaturgie, Bildführung, Charakter- und Rolleneigenschaften und das Storytelling insgesamt. Die klassische Filmsprache, die Inszenierung von Charakterzügen und personalen Entwicklungen im Film werden immer stärker professionell auf das Medium der Videospiele übertragen. Die Geschichte und Struktur des Spiels „Black Ops II“ (Activision, 2012) wurde etwa vom Drehbuchautoren David S. Goyer ${ }^{5}$ verfasst (Jenkins 2012). Hier verbinden sich die wirkmächtigsten Werkzeuge Hollywoods mit der Möglichkeit für den Spieler, sich durch die Vielzahl der interaktiven Möglichkeiten realitätsnah einer Handlung auszusetzen und diese zu beeinflussen. 
Auch moderne Videospiele erfüllen in der Konstruktion ihrer Hintergrundgeschichten und Sagenwelten nach wie vor die Prinzipien der griechischen Tragödien und schreiben die Traditionen von Shakespeares Königsdramen fort (vgl. Murray 1997, S. 27ff.): Komplexe Gesellschaftsstrukturen werden durch Protagonisten und Antagonisten vermittelt. Bedrohungsszenarien machen das Auftreten eines Helden notwendig, eine Welt muss vor dem Bösen gerettet werden. Das Spielziel steht oft aber so wenig fest wie der Weg dorthin. Beides benötigt den Input von Spielern. Da allerdings im Spiel mehr Eigenaktion nötig ist als im bloß passiv erfahrbaren Schauspiel oder dem Film, werden einzelne Handlungsstränge oder auch Charaktere weniger stark erinnert als im Film, der die distanzierte und evaluierende Beobachtung erlaubt, für die im von ständig notwendiger Aktion und Reaktion getriebenen Videospiel hingegen weder Zeit noch Raum bleiben. Zudem sind für Spieledesigner der Moment und die Umstände, wann Spieler einen NPC-Charakter (non player character - computergesteuert) wieder treffen, kaum abschätzbar. Eine zu große Zahl einander widersprechender Charaktere und Figuren dürfte zumindest momentan noch kaum einem Spieler vermittelbar sein.

Handlungsmotivation erschließt sich im Spiel deutlich mehrdimensionaler als im Film, aber sie setzt ebenso auf etabliertes Alltagswissen, das dem Publikum nicht mehr erklärt werden muss. Für die Betrachtung religiöser Elemente in Videospielen ist dies deshalb von Bedeutung, weil religiöse (und ideologische) Motive aller Art heute nicht mehr nur als Elemente der Hintergrundgeschichte benutzt und grafisch dargestellt werden können, sondern weil durch Filmsprache und interaktive Eingreifmöglichkeit mit technischer bzw. taktiler Unterstützung (z. B. vibrierende Joypads) subtile Vermittlungs- und auch Beeinflussungstechniken möglich sind. „LA Noire“ (Rockstar, 2011), ein Spiel der Produzenten der „Grand Theft Auto“-Serie, verlangt von Spielenden, dass sie vermittels lebensechter $\mathrm{Ge}$ sichtsmimik Spielecharaktere beim Lügen erwischen (vgl. Ernst 2011). „Spec Ops: The Line“ (2K Games, 2012) stellt die Spieler in einem militärischen Shooter sogar vor die moralische Frage, wie sie mit Gefangenen umgehen wollen (vgl. Frick 2012, Wüllner 2012). Will man die Adressierung ethischer Fragestellungen im Videospiel nicht bereits in der Lebenssimulation „Alter Ego“ (Activision, 1985) erkennen, so ist sie spätestens heute nicht mehr zu übersehen.

In digitalen Spielumgebungen können Werte und Ansichten heute also ebenso glaubwürdig wie in Büchern und Filmen 
vermittelt und vor allem nach- und miterlebt werden. Durch die riesigen und detaillierten Spielwelten sind seit einigen Jahren Entfaltungsmöglichkeiten realisierbar, die alternativen Lebensentwürfen gleichkommen. ${ }^{6}$ Durch die große Zahl an Möglichkeiten bilden sich die Transfers (in die Spielwelt hinein und aus dieser heraus) derart vielfältig aus, dass die Grenzen zwischen Spiel und Wirklichkeit verschwimmen und gerade durch das Spielen Online und die Kommunikation mit virtuellen Communities (und ihren realen Mitgliedern) in Echtzeit die Formen realen und interaktiven Erlebens und Erinnerns nach einiger Zeit ineinander übergehen. Das Gruppen- oder Clan-Spiel ist zum sozialen Event mit Wettbewerbscharakter geworden.

Der seit den Anfängen des Videospiels in den späten 1970erJahren immer wieder vorgebrachte Einwurf, sie vereinsamten deren Benutzer ${ }^{7}$, kann heute vor dem Hintergrund der Einbindung vieler Spiele in Funktionsumgebungen sozialer Netzwerke nicht mehr aufrecht erhalten werden. Schon aus demografischen Gründen ist die Zahl der direkten Spiel- oder Kommunikationspartner eines Jugendlichen

Sozialkontakte in der virtuellen Realität von Computerspielen anzustreben, ist heute eher Erweiterung und nicht Verengung sozialen Erlebens. im Wohnumfeld häufig gesunken (vgl. Kirchenamt der EKD 2010, S. 16ff.). Sozialkontakte in der virtuellen Realität anzustreben und sie wie selbstverständlich mit dem Spielen von Videospielen zu verbinden, ist heute eher Erweiterung und nicht Verengung sozialen Erlebens.

Für die meisten Spieler steht nach wie vor der Wettbewerb (vgl. Hartmann 2008, S. 211 ff.) und das Ringen mit dem Computer im Zentrum, während Außenstehende sich auf gewalttätige grafische Darstellungen konzentrieren. Kirchen versuchen

6 In "Grand Theft Auto: San Andreas" (Rockstar Games / Take2, 2004), das eine komplett begehbare Welt mit drei großen Städten, dutzenden Dörfern und Wüsten- und Waldlandschaften bietet, konnten Spieler schon vor zehn Jahren losgelöst und frei von der komplexen Missionsstruktur frei agieren und beispielsweise Fitnessübungen durchführen, um ihre Körperfunktionen zu verbessern oder auch länger tauchen zu können, sie konnten sich ungesund oder auch bewusst ernähren und Kraft gewinnen sowie ihre verschiedenen Fähigkeiten trainieren. Sie konnten Helikopter fliegen, Boot und Eisenbahn fahren, Trucks steuern und Waren transportieren und dergleichen mehr - all dies außerhalb jeder Mission und rein zum Spaß.

7 So noch Grunder 2001, S. 110. Er beachtet die Literaturlage hierzu leider nicht. 
mittlerweile alternative Angebote (vgl. EKD 2014) zu machen und sich verstärkt um Differenzierung zu bemühen. ${ }^{8}$

\section{(Mangelndes) Interesse der Religionspädagogik an Spielen und Entwicklern}

Videospiele enthalten vielfältige religiöse Mythologeme, die sich zumal nicht auf eine Konfession begrenzen lassen. Heilssymbole wie die altägyptische Lebenshieroglyphe „Anch“ finden sich im Spiel oder auf Titelillustrationen wie selbstverständlich neben anderen. ${ }^{9}$ Esoterische und magische Einflüsse sind ebenso enthalten wie religiöse Moralvorstellungen, die dazu dienen, innerhalb der Onlinewelt Wohlverhalten zu begründen und soziale Kontrolle aufrecht zu erhalten. Dass die öffentlich wirksamen Stereotype der Gewalthaltigkeit und des Suchtpotentials von Videospielen nach wie vor die öffentliche Würdigung des Mediums dominieren, zählt Winklmann zu den Gründen dafür, dass die positiven Möglichkeiten und Potentiale für Bildungsprozesse oft „unter den Tisch“ fallen (2014, S. 52).

Bildung zu vermitteln, Identität zu schärfen und Weltsichten $\mathrm{zu}$ weiten und $\mathrm{zu}$ fundieren sind traditionelle Aufgaben der Religionspädagogik. Die entscheidende religionspädagogische Aufgabe, nämlich auch danach zu fragen und

Esoterische und magische Einflüsse sind in Videospielen ebenso enthalten wie religiöse Moralvorstellungen, die dazu dienen, Wohlverhalten zu begründen. sich dafür zu interessieren, ob mediale Verarbeitungen von Religion den Individuen Raum lassen bzw. geben, sich kritisch, würdigend oder distanzierend $\mathrm{zu}$ ihnen $\mathrm{zu}$ verhalten (vgl. Schröder 2012, S. 226), darf aber die Anbieter und Erschaffer von derartigen Inhalten nicht ausblenden. Viele Aus- und Rückwirkungen virtuell-spielenden und erlebenden Handelns sind nicht rein durch die Beobachtung vermittelbar, sondern müssen tatsächlich auch selbst erlebt werden. Zu bedenken geben Burris und Dow, dass Videospiele durch das, was sie zeigen, auch die Vorstellungskraft der Nutzenden verändern können. Auf Basis ihrer Studie „Lost in the Myst“ schlussfolgern sie, dass die visuelle Realität durch die schiere Prägnanz ihrer Bilder wenig Raum für Vorstellungen lasse und sich dadurch auch verringernd auf religiöses Interesse auswirken könne (vgl. Burris/Dow 2014).

8 Schleider 2009: Die Kommentare der Leser auf die vorgestellte kirchliche Initiative lassen aber auf wenig religiöses Interesse schließen.

9 Frühe Beispiele: das Adventure „Raiders of the lost Ark“, Atari (1982) oder das Rollenspiel „Ultima IV“, Origin Systems Inc. (1985). 
Zuvor hatte Burris bereits mit Redden untersucht, inwieweit Atheisten und Religiöse unterschiedlich mit Emotionen umgingen, wie sie beispielsweise auf tragische Nachrichtenmeldungen oder die Erinnerung an eine Liebesgeschichte reagierten und ob Videospiele eigentlich attraktiver für Atheisten oder für Religiöse wären (Burris/Redden 2012, S. 243f.) - möglicherweise, weil sie die Vorstellungskraft anders berührten und forderten als Brettspiele. Meiden Atheisten möglicherweise sogar Aktivitäten, die Vorstellungskraft voraussetzen (ebd., S. 249)?

Gemäß ihrer Ergebnisse bevorzugten Atheisten eher Videospiele als andere (Religiöse / Agnostiker / nicht-Religiöse), die ihrerseits tendenziell eher Brettspiele mochten. Die Differenzen in der Spielepräferenz führen die Autoren auf neuronale Unterschiede zurück ${ }^{10}$, die zu verschiedenen Imaginationsfähigkeiten und -vorlieben führten und damit das Interesse an Spielen erklärten, die einer gewissen Vorstellungskraft bedürften (vgl. Burris/ Redden 2012, S. 250). Wie Brettspiele, so schlussfolgern sie, handele auch Religion vom „Was wäre wenn“-Handeln und die Existenz neuronaler Unterschiede ließe es verständlich erscheinen, dass gemäß der bestehenden biologischen Voraussetzungen und der persönlichen Überzeugungen „unsichtbare“ Spielbestandteile unterschiedlich akzeptiert und präferiert würden (ebd.).

Wie mühsam (aber auch interessant und wohl kurzweilig) es sein kann, wenn sich Gegner oder Kritiker von Videospielen pädagogisch auf diese einlassen, erläutert Scholtz anhand seiner eigenen Beschäftigung mit dem bekannten Konsolenspiel „Zelda“ (2004, S. 145ff.). ${ }^{11}$ Eher unfreiwillig bestätigt er viele Vorurteile auch von Spielern selbst gegenüber Älteren (und damit den bereits eingangs genannten erziehenden oder pädagogischen Beobachtenden), wenn er etwa schreibt, dass er als Religionspädagoge „gegen seine sonstigen Gewohnheiten selbst spielt, wenn er nicht nur Zuschauer ist, sondern sich selbst ins Spiel bringt und aufs Spiel setzt" (ebd., S. 146) und so seine eigenen Stereotypen demaskiert. Er zeichnet seinen evolutionären Verständnisprozess nach: Während er zu Beginn aus der Schwierigkeit des Spiels für ungeübte Erwachsene den Beleg für ein „Differenzphänomen“ von Jugendkultur konstruiert (ebd., S.147) (wobei man auch

10 Hierzu und zur „Willensfreiheit“ insbesondere auch: Becker 2009, S. 41 und S. 202.

11 An dieser Stelle muss darauf hingewiesen werden, dass "Zelda“ eben kein Computerspiel ist, wie im Titel angegeben, sondern ein Konsolenspiel. 
von Ungeübtheit aufgrund seines bisherigen Desinteresses sprechen könnte) und „abgrenzende Funktionen“ sowie eine „herzustellende Differenz“ auch anhand der „Inhalte der Computerspiele durch Gewalt oder politische Radikalität der Abgrenzung und Provokation“ (ebd.) ${ }^{12}$ identifiziert, erfährt Scholtz selbst mit zunehmender Beschäftigung den Reiz des Spiels und erlebt sowohl Flow wie auch die Auswirkungen immersiver Annäherung (ebd.). Hiervon profitiert er, denn nun, und zwar erst jetzt, fallen ihm die „explizit religiösen Zitate“ auf, die das Spiel mithilfe von Symbolen und Elementen aus unterschiedlichen religiösen und mythologischen Traditionen in den Geschichten und der Sprache von „Zelda“ verwendet und erzeugt (ebd., S. 148).

Sind Spiele also letztlich Träger oder Vermittler von Religion? Überraschenderweise gesellt sich Scholtz argumentativ zu Lange (ebd.), welcher zuvor konstatiert hatte, dass die Hintergrundgeschichten von Spielen keine wesentliche Bedeutung für das Spielen selbst hätten (vgl. 2001, S. 79ff.). Tatsächlich behauptete Lange, dass überdimensionierte Hintergrundgeschichten eigentlich nur die abstrakten Regelmuster von Videospielen verbergen sollten (ebd., S. 79). Er möchte „den Fokus auf die zerstörerischen Kräfte lenken, die Games im Hinblick auf die in ihnen angeblich erzählten Geschichten innewohnen (ebd., S. 80)“. Inhaltlich begründet Lange seinen Verdacht des destruktiven Potentials von Spielen mit zu dünnen Hintergrundgeschichten, die den Möglichkeiten des Mediums nicht gerecht würden und die sich ausgiebig und schamlos aus einem gesellschaftlichen kulturellen Fundus bedienten (ebd.). Diese Behauptung lässt sich weder inhaltlich noch quantitativ aufrechterhalten und zudem zeigen etliche Foren im Internet, dass viele Spieler die Hintergrundgeschichten sehr schätzen und verehren und sogar eigenhändig die Plots fortschreiben (Fan-Fiction).

Scholtz findet mit seinem phänomenologischen Ansatz jedenfalls zu neuen Einsichten über Videospiele, die er durch das reine Beobachten zuvor nicht hatte (vgl. 2004, S. 151) und kommt zu dem Schluss, „dass im Umgang mit Computerspielen eine fiktive Welt für bedeutsam gehalten wird und dass sich daraus ein Gegenpol zu dem immer stärker dominierenden, rein am Materiellen und an naturwissenschaftlichen Parametern orien- 
tierten Wirklichkeitsverständnis bilden kann. Solche strukturellen Analogien zur religiösen Weltkonzeption können genauso wie der im Umgang mit Computerspielen geübte Symbolumgang, die Einübung imaginativer Prozesse und der Wechsel zwischen verschiedenen Realitätsebenen zur Stärkung religiöser Kompetenz führen“ (ebd., S. 150f.).

Der Medienpädagogik kann mit Brüggen (nach Schell) vorgeworfen werden, dass man sich zwar einerseits für aktive Medienarbeit stark mache (vgl. Schell 2003, S. 30), die Medienpädagogik sei aber „unmächtig“ und „nicht bei Bewusstsein“, wenn sie auf bedenkliche Entwicklungen nur durch ansonsten kritische Untätigkeit reagiere (ebd., S. 1150f.). Dieser Befund kann auf die Religionspädagogik übertragen werden. Auch kirchenferne Kinder wachsen - schon durch Videospielwelten - nicht religionsfrei

Es müsste noch stärker nach den religiösen Sinngehalten gefragt werden, die von Unterhaltungsprogrammen ausgehen. auf $^{13}$, weshalb die Mediensozialisation von Menschen gerade auch für die Religionspädagogik eine Verpflichtung darstellt, sich nicht nur um Oberflächenphänomene wie Gewalt, sondern auch ganz grundsätzlich mit Inhalten und ihren Erzeugern zu beschäftigen.

Gräb zufolge müsste noch stärker nach den religiösen Sinngehalten gefragt werden, die von Unterhaltungsprogrammen ausgehen - denn ob religiöse Kodierungen vorgenommen und symbolische Vermittlungen angeboten und diese von Rezipientinnen und Rezipienten überhaupt bewusst für die Sinnorientierung aufgegriffen werden (können), wäre sonst kaum sichtbar zu machen (vgl. Gräb 2006, S. 23f.). Er begrüßt, dass das Gewicht der Rezipientenperspektive in der Forschung zugenommen hat und wünscht eine „religionssensible Medienrezeptionsforschung“ (ebd., S. 26). Hinzuzufügen wäre nun, dass aber vor allem auch eine religionssensible Kommunikatorenforschung zu leisten ist, die sichtbar macht, wer welche religiösen Inhalte in Unterhaltungsprogrammen platziert. Dies ist bislang ausgeblieben.

13 „Entgegen einer einseitigen Orientierung an der Säkularisierungsthese wäre demnach davon auszugehen, dass auch kirchenferne junge Menschen in unserer Zeit nicht "religionsfrei" aufwachsen; sie sind, was Religion betrifft, keine unbeschriebenen Blätter. [...] Medienerziehung bzw. Medienbildung wäre dann also nicht eine Aufgabe, die gleichsam von außen an die religiöse Erziehung und Bildung herangetragen wird, sondern sie stellte sich vielmehr als genuin religionspädagogische Notwendigkeit dar" (Pirner 2004, S. 154f.). 
Den Forschungsstand zu der Frage, wie Medien und insbesondere Computer- und Videospiele die religiöse Sozialisation von Kindern und Jugendlichen beeinflussen, hat Pirner noch 2012 (vgl. 2012a, S. 67) als unbefriedigend bezeichnen müssen. Und noch immer fehlen Studien zu den Personen und ihren Intentionen, die für diese Medienwelten verantwortlich sind!

\section{Sind Spieleentwickler unreligiös?}

Bislang existieren kaum Untersuchungen zu der Frage, wie Spieleentwickler weltanschaulich empfinden und ob möglicherweise ihre religiösen oder politischen Ansichten in ihre Produktionen einfließen. Eine internationale Befragung ergab 2005 (vgl. IGDA 2005, S. 15), dass manche Entwickler auf Fragen nach ihrer sexuellen Ausrichtung extrem ablehnend reagierten. Die damalige Untersuchung schloss ausdrücklich keine weltanschaulichen Ansichten mit ein. Eine vom Autoren 2012 an der Berliner Games Academy durchgeführte Befragung von 59 jungen Spieleentwicklerinnen und -entwicklern ergab ähnlich heftige Abwehrreaktionen hinsichtlich weltanschaulicher Positionen (Piasecki, Veröffentlichung in Vorbereitung).

Die allermeisten der dort befragten angehenden Spieleentwickler haben keine religiöse Sozialisation erfahren: Bei zwei Drittel der Befragten wurde zuhause nie gebetet („Niemals“: 67,2 Prozent), nur etwa jeder Zehnte geht zu einem bestimmten Anlass oder häufiger in einen Gottesdienst (Gottesdienstbesuch „Nie“: 88,9 Prozent). Mehr als die Hälfte glaubt gar nicht an Gott (Gottesglauben „Nein“: 55,6 Prozent, „Eher nicht“: 9,3 Prozent, „Ausgeglichen“: 16,7 Prozent, „Sicher ist da etwas“: 9,3 Prozent, „Ganz klar, ich glaube an Gott“: 3,7 Prozent, „Keine Angabe“: 5,6 Prozent). Eher oder deutlich zustimmend bei der Frage nach dem persönlichen Gottesglauben äußern sich damit weniger als 15 Prozent. Einer der christlichen Konfessionen gehören jedoch nach wie vor mit 53,6 Prozent mehr als die Hälfte der Befragten („Römisch-Katholisch“: 14,6 Prozent, „Evangelisch“: 39 Prozent) an, 39 Prozent sind aus einer Konfession ausgetreten. Andere als christliche organisierte Konfessionen waren unter den Befragten nicht vorhanden.

Ein persönliches Interesse an religiösen Themen verspürt ebenfalls nur eine Minderheit der Befragten: Insgesamt 8,8 Prozent haben „starkes“ oder „mäßig starkes Interesse“ („Kein Interesse“: 47,4 Prozent, „Schwaches Interesse“: 28,1 Prozent, „Ausgeglichen“: 15,8 Prozent). Gleichzeitig können sie sich die Integration von Religion in ein Videospiel vorstellen, wenn dies 
mit dem Spielziel oder dem Spielinhalt begründet ist („Definitiv ja“: 7,7 Prozent, „Warum nicht“: 30,8 Prozent, „Nein, auf keinen Fall“: 3,8 Prozent, „Wenn die Geschichte es hergibt“: 21,2 Prozent, „Wenn das Spielsetting es verlangt“: 23,1 Prozent, „Nur als Mittel im Spiel (Magiequelle, Gegnermacht)“: 13,5 Prozent). Das klassische Gottesbild wird dabei nicht favorisiert, sondern Gott im Spiel eher in Form der Existenz eines „höheren Wesens“ oder einer „kosmischen Energie“ gesehen.

Die Frage nach der Vermittelbarkeit sozialer und gesellschaftlicher Themen in Videospielen (Behinderung, gleichgeschlechtliche Liebe, soziale Ausgrenzung etc.) wird ganz eindeutig bejaht. 62,7 Prozent der Befragten halten gesellschaftliche Themen für „absolut umsetzbar“. Noch 23,5 Prozent halten dies vorsichtig für "machbar“. „Unschlüssig“ sind 5,9 Prozent, für "schwer, kaum machbar" halten es 7,8 Prozent. Niemand glaubt, dass dies gar nicht realisierbar sei. Spieleentwickler scheinen grundsätzlich interessiert an gesellschaftlichen Themen - nahezu die Hälfte zeigt sogar ein starkes (24,1 Prozent) oder noch mäßig starkes Interesse Das klassische Gottesbild wird von (24,1 Prozent) an Fragen der Gerechtigkeit, von Krieg und Frieden oder auch der Umwelt Spielerentwicklern nicht favorisiert. Es geht um die Existenz eines höheren („Ausgeglichen“: 29,3 Prozent, „Schwaches Wesens oder einer kosmischen Energie. Interesse“: 17,2 Prozent, „Kein Interesse“:

5,2 Prozent). Allerdings ist insgesamt nur weniger als ein Fünftel entweder politisch („Überwiegend politisch tätig“: 5,5 Prozent) oder gesellschaftlich („Ich übe überwiegend ein gesellschaftliches Ehrenamt aus“: 10,9 Prozent) engagiert („Ehrenamtlich oder politisch engagiert: Nein“: 83,6 Prozent). Mehr als die Hälfte der Befragten sind der Meinung, dass Fragen von großer gesellschaftlicher oder kultureller Bedeutung in Spielen unterhaltsam und lehrreich thematisiert werden können: 37,7 Prozent sind davon "absolut" überzeugt, „eher überzeugt" sind 11,3 Prozent, über ein Viertel (26,4 Prozent) sagen, dass dies abhängig vom Spieldesign zu realisieren sei („Eher nicht“: 13,2 Prozent, „Nein“: 11,3 Prozent).

Bedeutend ist allerdings die zuvor auch bei Journalisten (Schneider/Schönbach/Stürzebecher 1993, S. 20 ff.) immer wieder beobachtete und grundsätzlich vorhandene Bereitschaft, die eigenen Positionen und selbst vertretenen Meinungen und Überzeugungen in die bearbeiteten Spiele zu transferieren. Nur eine Minderheit lehnt dies ab („Nie“ 2 Prozent, „Selten“ 9,8 Prozent). Nahezu die Hälfte („Manchmal“ 39,2 Prozent, „Häufig“ 37,3 Prozent, „Immer“ 9,8 Prozent) kann sich vorstellen, dies auf 
jeden Fall oder häufig zu tun und Inhalte von Spielen gemäß den eigenen persönlichen Überzeugungen zu gestalten. ${ }^{14}$

\section{Resümee}

Wer als Spielender eine virtuelle Welt betritt, wird mit religiösen Elementen konfrontiert (vgl. Kurth 2009), die zum Bestandteil der individuellen Spielhandlung werden - ob persönlich gläubig oder ungläubig. Religion ist Teil des Unterhaltungsinstrumentariums; Mitmachen ist nicht nur erwünscht, sondern zum (virtuellen) Überleben wichtig. Spieleentwicklerinnen und -entwickler, als Medienerschaffer und Kommunikatoren bislang nicht untersucht, konnten in der Befragung mehrheitlich zwar (in Anlehnung an Max Weber) als „religiös unmusikalisch“ identifiziert

Entwickler sind bereit, Spielinhalte mit eigenen Überzeugungen "aufzuladen" und Religion als Narrationsbestandteil zu instrumentalisieren. werden, gleichzeitig sind sie allerdings bereit, Spielinhalte mit ihren eigenen Überzeugungen "aufzuladen“ und Religion als Narrationsbestandteil $\mathrm{zu}$ instrumentalisieren. $\mathrm{Zu}$ beachten ist, dass sich Mitmachmedien wie Videospiele nicht nur zu Unterhaltungszwecken einsetzen lassen: Sie ermöglichen Kirchen genauso eine interaktive Mission wie sie fundamentalistischen Gewalttätern eine virtuelle Dschihad-Plattform bieten können. Mit dem Spiel "Special Force" hat die libanesische Hisbollah 2003 ein solches Spiel vorgelegt, das mittlerweile allerdings technisch überholt ist (Piasecki 2003, S. 42). Soziale Netzwerke und virtuelle Lernräume sind seitdem für die Vermittlung auch extremistischer Botschaften allerdings wesentlich bedeutsamer geworden.

Den Glauben an die gerechte Sache kann der Videospieler von heute sehen, ausprobieren und praktizieren. Er kann sympathisch gezeichneten Charakteren nacheifern und sich von ihnen in ihre Welt einführen und für die richtige Sache begeistern lassen. Er kann mit geschundenen und benachteiligten Charakteren stärker mitleiden als im Film, denn hier ist er nicht auf die passive Rezeption beschränkt; er kann eingreifen und angreifen. Während die virtuelle Gemeinde ihm dafür Lob und Anerkennung zollt, bekommt das reale Umfeld dies aber unter Umständen nicht mit. Einer religiösen oder politischen Radikalisierung eines im Spiel kämpfenden Jugendlichen müssen demzufolge 
nicht einmal die engsten Verwandten gewahr werden. Es gibt daher etliche Gründe, nicht nur weiterhin reine Rezeptionsforschung zu betreiben, sondern auch aktiv nach Intentionen und Vorstellungen der Medienerschaffer und ihren weltanschaulichen und ethischen Grundeinstellungen zu fragen.

\section{Zentrale Befunde}

- Videospiele sind nie ein grundsätzlich gültiges Modell der Wirklichkeit, sie bieten allerdings glaubwürdige Realitätsbestandteile innerhalb ihrer eigenen Konventionen.

- Computer- und Videospiele sind kein reines Jugendphänomen mehr. Die Zahl älterer Nutzer wächst rapide. Sie werden nach neuen Themen verlangen.

- Spielelemente greifen über in soziale Netzwerke und virtuelle sowie marktwirtschaftliche Prozesse. Probehandeln ist kein Gegenstand der reinen Unterhaltung mehr.

- Das Videospiel kann ein Proberaum zum Austesten von Denk- und Verhaltensmodellen sein.

- Spiele sind lehrreich, weil man tatsächlich aktiv lernen muss, um sie zu bestehen.

- Videospiele sind keine autonome Parallelwelt. Sie blockieren aber Lebenszeit und sind damit eine Konkurrenz für alle anderen Bereiche (Bildung, Ehrenamt etc.) und Optionen des Lebens, die in der gleichen Zeit nicht stattfinden können.

- Spiele wie auch Medien allgemein begleiten und initiieren Selbstsozialisierungsprozesse.

- Entwickler haben große Verantwortung, indem sie Grenzen und Möglichkeiten von Weltvermittlung und -darstellung sowie -rezeption schaffen und das Erleben der Spielenden rahmen und führen.

- Religion ebenso wie ethische Implikationen im Videospiel erscheinen vor diesem Hintergrund als bislang zu Unrecht vernachlässigt und es gilt so für die Religionspädagogik, was Böhm und Buschmann hinsichtlich der Anerkennung von Popmusik formuliert haben: „Religionspädagogik hat besonders dann eine Zukunft, wenn sie ihren vielfach erheblichen, häufig überheblichen Analphabetismus im Hinblick auf die Lebenswelten von Kindern und Jugendlichen überwindet und diese stärker als bisher in ihre Überlegungen einbezieht (Böhm/Buschmann 2000, S. 11).“

- Aus den Erfahrungen von Scholtz ist außerdem zu lernen: Sprachfähig wird nicht, wer von außen zuschaut und kritisiert. Urteilsfähig werden kritisch Spielende. 


\section{Literatur}

Alexander, Constantin (2013): Experte: Internetsucht nimmt zu. http://www. heise.de/newsticker/meldung/Experte-Internetsucht-nimmt-zu-1776726. html (zuletzt aufgerufen am 14.3.2014).

Baudrillard, Jean (2000): Der unmögliche Tausch. Berlin.

Becker, Patrick (2009): In der Bewusstseinsfalle? Geist und Gehirn in der Diskussion von Theologie, Philosophie und Naturwissenschaften. Göttingen.

Bevc, Tobias (Hg.) (2007): Computerspiele und Politik. Zur Konstruktion von Politik und Gesellschaft in Computerspielen. Münster.

Böhm, Uwe/Buschmann, Gerd (2000): Popmusik - Religion - Unterricht: Modelle und Materialien zur Didaktik von Popularkultur. Münster.

Bornhöft, Petra et al. (2009): 113 Kugeln kalte Wut. In: Spiegel, H. 12, S. 3046. http://www.spiegel.de/spiegel/print/d-64628264.html (zuletzt aufgerufen am 17.7.2014).

Brenner, Christian (2003): Der Computer als Medium im Religionsunterricht. Münster.

Brüggen, Niels (2014): Wer ist hier der Souverän? Kritische Anmerkungen zur Medienkompetenzförderung in der digitalen Gesellschaft. In: merz medien + erziehung, 58. Jg., H.1, S. 28-35.

Burris, Christopher T./Redden, Elyse K. (2012): No Other Gods Before Mario?: Game Preferences Among Atheistic and Religious Individuals. In: International Journal for the Psychology of Religion, 22. Jg., S. 243-251.

Burris, Christopher T./Dow, Traci (2014): Lost in the Myst?: Narrative Video Gaming Decreases Self-Reported Propensity for Spiritual / Religious Experience. Abstract. In: International Journal for the Psychology of Religion, 24. Jg., Published Online 23.1.2014, http://www.tandfonline.com/doi/ful l/10.1080/10508619.2014.884393\#.UuyUICjyits (zuletzt aufgerufen am 10.5.2014).

Dinter, Astrid (2007): Adoleszenz und Computer. Von Bildungsprozessen und religiöser Valenz. Göttingen.

Dogruel, Leyla (2008): Computerspiele und 50+. Akzeptanz und Potentiale von Computerspielen bei Personen ab 50 Jahren. München.

EKD (Hg.): Spiele. http://www.ekd.de/spiele/44280.html (zuletzt aufgerufen am 14.3.2014).

Englert, Rudolf et al. (Hg.) (2012): Gott googeln? Multimedia und Religion. Jahrbuch der Religionspädagogik (JRP 28). Neukirchen-Vluyn.

Ernst, Nina (2011): Diese Augen können lügen. http://www.stern.de/digital/ spiele/krimi-videospiel-la-noire-diese-augen-koennen-luegen-1687208.html (zuletzt aufgerufen am 14.3.2014).

Freudenberger-Lötz, Petra (72012): Schüler/in - theologisch. In: Rothgangel, Martin/Adam, Gottfried/Lachmann, Rainer (Hg.): Religionspädagogisches Kompendium. Göttingen, S. 252-264. 
Freyermuth, Gundolf S./Gotto, Lisa/Wallenfels, Fabian (Hg.) (2013): Serious Games Exergames Exerlearning. Zur Transmedialisierung und Gamification des Wissenstransfers. Bielefeld.

Frick, Daniel (2012): Spiele machen Ernst. In: pro-Cristliches Medienmagazin, H.5, S. 44-45. http://www.pro-medienmagazin.de/uploads/tx_booklet/ PRO_2012_05.pdf (zuletzt aufgerufen am 2.9.2014).

Göttlich, Udo/Krotz, Friedrich/Paus-Haase, Ingrid (Hg.) (2001): Daily Soaps und Daily Talks im Alltag von Jugendlichen. Opladen.

Gräb, Wilhelm et al. (2006): „Irgendwie fühl ich mich wie Frodo...!" Eine empirische Studie zum Phänomen der Medienreligion. Frankfurt am Main.

Gräb, Wilhelm/Herrmann, Jörg (2006): Gelebte Medienreligion. In: Gräb, Wilhelm et al.: "Irgendwie fühl ich mich wie Frodo...!" Eine empirische Studie zum Phänomen der Medienreligion. Frankfurt am Main, S. 7-14.

Grethlein, Christian (1998): Religionspädagogik. Berlin.

Grümme, Bernhard/Lenhard, Hartmut/Pirner, Manfred L. (Hg.) (2012): Religionsunterricht neu denken. Innovative Ansätze und Perspektiven für den Religionsunterricht. Stuttgart.

Grunder, Hans-Ulrich (2001): Schule und Lebenswelt. Ein Studienbuch. Münster. Hartmann, Maren/Hepp, Andreas (Hg.) (2010): Die Mediatisierung der Alltagswelt. Wiesbaden.

Hartmann, Thilo (2008): Let's compete! Wer nutzt den sozialen Wettbewerb in Computerspielen. In: Quandt, Thorsten/Wimmer, Jeffrey/Wolling, Jens (Hg.): Die Computerspieler. Studien zur Nutzung von Computergames. Wiesbaden, S. 193-208.

Heumann, Jürgen (Hg.) (2005): Über Gott und die Welt. Religion, Sinn und Werte im Kinder- und Jugendbuch. Frankfurt am Main.

Höfner, Charlotte (2003): Sind Nachrichtenfaktoren Unterhaltungsfaktoren? Eine experimentelle Überprüfung des Einflusses von Nachrichtenfaktoren auf den wahrgenommenen Unterhaltungswert und die wahrgenommene Informationsqualität von politischen Zeitungsmeldungen. Diss. Univ. München.

IGDA (International Game Developers Association) (Hg.) (2005): Game Developer Demographics: An Exploration of Workforce Diversity. http://www. igda.org/sites/default/files/IGDA_DeveloperDemographics_Oct05.pdf (zuletzt aufgerufen am 16.6.2014).

Jenkins, David (2012): David S. Goyer Black Ops II interview - the white knight of storytelling. http://metro.co.uk/2012/11/13/david-s-goyer-blackops-ii-interview-the-white-knight-of-storytelling-618510/ (zuletzt aufgerufen am 14.2.2014).

Keppler, Angela (2010): Variationen des Selbstverständnisses: Das Fernsehen als Schauplatz der Formung sozialer Identität. In: Hartmann, Maren/ Hepp, Andreas (Hg.): Die Mediatisierung der Alltagswelt. Wiesbaden, S. 111-126. 
Kirchenamt der EKD (Hg.) (2010): Kirche und Jugend - Lebenslagen, Begegnungsfelder, Perspektiven. Eine Handreichung des Rates der Evangelischen Kirche in Deutschland. Gütersloh.

Konz, Britta (2005): Virtualisierung menschlicher Erfahrung. Menschenbild im Videospiel. In: Heumann, Jürgen (Hg.): Über Gott und die Welt. Religion, Sinn und Werte im Kinder- und Jugendbuch. Frankfurt am Main, S. 237-258.

Kopka, Tobias (2013): Interface Control Meaning. Eine typologische Gegenstandssichtung des Phänomens Exergames. In: Freyermuth, Gundolf S./ Gotto, Lisa/Wallenfels, Fabian (Hg.): Serious Games Exergames Exerlearning. Zur Transmedialisierung und Gamification des Wissenstransfers. Bielefeld, S. 265-288.

Kurth, Cornelia (2009): „Wie gläubig man in einem Spiel doch sein kann!” In: evangelisch.de vom 23.11.2009 http://www2.evangelisch.de/themen/medien/wie-glaeubig-man-in-einem-spiel-doch-sein-kann7254 (zuletzt aufgerufen am 14.3.2014).

Kreusing, Bastian (2014): Computerspielsucht erkennen und verstehen. http:// www.spieleratgeber-nrw.de/?siteid=1369 (aufgerufen am 14.3.2014).

Lange, Andreas (2001): Storykiller. Von der Zerstörung der Geschichten in Computerspielen. In: Ästhetik \& Kommunikation, 32.Jg., H. 115, S. 79-84.

Meister, Dorothee M. et al. (2008): Mediale Gewalt. Ihre Rezeption, Wahrnehmung und Bewertung durch Jugendliche. Wiesbaden.

Murray, Janet H. (1997): Hamlet on the Holodeck. The Future of Narrative in Cyberspace. New York.

Nord, Ilona (2008): Realitäten des Glaubens. Zur virtuellen Dimension christlicher Religiosität. Berlin.

Paus-Haase, Ingrid et al. (1999): Talkshows im Alltag von Jugendlichen. Opladen.

Piasecki, Stefan (2003): Für 8,50 Dollar ziehen sie in den Krieg gegen Israel. In: Frankfurter Allgemeine Zeitung vom 20.11., S. 42.

Piasecki, Stefan (2009): Religion in der Mediengesellschaft - Verständnisse und Missverständnisse. Marburg.

Piasecki, Stefan: Erlösung durch Vernichtung ?! - Unterhaltung, Technik-Faszination und Muster des Religiösen im Videospiel. Eine explorative Studie an Schnittpunkten von Religionspädagogik, Sozialwissenschaften und Game Studies. Veröffentlichung in Vorbereitung.

Pirner, Manfred L./Breuer, Thomas (Hg.) (2004): Medien - Bildung - Religion. Zum Verhältnis von Medienpädagogik und Religionspädagogik in Theorie, Empirie und Praxis. München.

Pirner, Manfred L. (2004): „Religiöse Mediensozialisation?" Ein empirisches Forschungsprojekt. In: Pirner, Manfred L./Breuer, Thomas (Hg.): Medien - Bildung - Religion. Zum Verhältnis von Medienpädagogik und Religionspädagogik in Theorie, Empirie und Praxis. München, S. 154-164. 
Pirner, Manfred L. (2012a): Religiöse Mediensozialisation. Wie die Medien die Religiosität von Kindern und Jugendlichen beeinflussen. In: Englert, Rudolf et al. (Hg.): Gott googeln? Multimedia und Religion. Jahrbuch der Religionspädagogik (JRP 28). Neukirchen-Vluyn, S. 59-69.

Pirner, Manfred L. (2012b): Medienweltorientierte Religionsdidaktik. In: Grümme, Bernhard/Lenhard, Hartmut/Pirner, Manfred L. (Hg.): Religionsunterricht neu denken. Innovative Ansätze und Perspektiven für den Religionsunterricht. Stuttgart, S. 159-172.

Quandt, Thorsten/Wimmer, Jeffrey/Wolling, Jens (Hg.) (2008): Die Computerspieler. Studien zur Nutzung von Computergames. Wiesbaden.

Reinders, Angela M. T. (2006): Zugänge und Analysen zur religiösen Dimension des Cyberspace. Münster.

Rothgangel, Martin/Adam, Gottfried/Lachmann, Rainer (Hg.) (2012): Religionspädagogisches Kompendium. Göttingen.

Schell, Fred (2003): Aktive Medienarbeit mit Jugendlichen. Theorie und Praxis. München.

Schleider, Thomas (2009): Evangelische Kirche: Computerspiele kein Teufelszeug. http://www.pcgameshardware.de/Spiele-Thema-239104/News/ Empfehlung-an-alle-Eltern-und-Grosseltern-701953/ (zuletzt aufgerufen am 14.3.2014).

Schneider, Beate/Schönbach, Klaus/Stürzebecher, Dieter (1993): Westdeutsche Journalisten im Vergleich: jung, professionell und mit Spaß bei der Arbeit. In Publizistik, 38. Jg., H.1, S. 5-30.

Scholtz, Christopher P. (2004): Religionspädagogisches Lernen in der Welt Zeldas? Phänomenologische Auseinandersetzung mit einem Klassiker der Computerspiele. In: Pirner, Manfred L./Breuer, Thomas (Hg.): Medien - Bildung - Religion Zum Verhältnis von Medienpädagogik und Religionspädagogik in Theorie, Empirie und Praxis. München, S. 145-153.

Schröder, Bernd (2012): Multimedia und Religion. Rückfragen - Kontroversen Perspektiven. In: Englert, Rudolf et al.(Hg.): Gott googeln? Multimedia und Religion. Jahrbuch der Religionspädagogik (JRP 28). Neukirchen-Vluyn, S. 221-233.

USK (Unterhaltungssoftware Selbstkontrolle)(Hg.): Genres. http://www.usk. de/pruefverfahren/genres/ (zuletzt aufgerufen am 14.3.2014).

Waltemathe, Michael (2011): Computer-Welten und Religion: Aspekte angemessenen Computergebrauchs in religiösen Lernprozessen. Hamburg.

Winklmann, Michael (2014): Biografie: Ein Spiel. Das identitätsbildende Potenzial von Computerspielen in (religiösen) Bildungsprozessen. In: merz medien + erziehung, 58. Jg., H.1, S. 52-57.

Wüllner, Daniel (2012): Bild: Spec Ops: The Line. In: Sueddeutsche.de vom 15.7. http://www.sueddeutsche.de/digital/spiele-test-spec-ops-the-lineim-herzen-der-eigenen-finsternis-1.1407897 (zuletzt aufgerufen am 14.3.2014). 